\title{
Wasser, Konflikte und soziales Kapital im Hohen Atlas Südmarokkos
}

\section{Olivier Graefe, Bayreuth}

\section{Konflikte um Wasser: Folge von Ressourcen- knappheit?}

Angesichts der Bevölkerungszunahme und der globalen Klimaveränderungen steigt die Besorgnis über die langfristige Wasserversorgung in semi-ariden und ariden Ländern. Vor allem in Nordafrika und dem Mittleren Orient, wo der durchschnittliche Wasserverbrauch pro Kopf wie auch die absolute Bevölkerungszahl zunehmen, verfügt jeder Einwohner dieser Region bereits seit dem Jahr 2000 im Durchschnitt über weniger als $1700 \mathrm{~m}^{3}$ pro Jahr (UNITED NATIONS Environmental Programme, UNEP 2002). Auch wenn die Folgen dieser Wasserverknappung auf der zwischenstaatlichen Ebene in Bezug auf das Kriegsund Konfliktpotential, unter anderem im Nahen Osten, unterschiedlich bewertet werden (vgl. AYEB 1998; Bulloch \& DarWISH 1993; LASSERRE 1999, 2003; Mutin 2000; Soffer 1999; Starr 1991), fundieren diese Analysen generell auf ressourcenzentrischen Ansätzen neomalthusianischer Prägung. Amartya Sen hat jedoch mit seinem Konzept der Verfügungsrechte in seiner bekannten Studie über die Ursachen des Hungers (1981) den Fokus auf die sozialen Aspekte der Ressourcenverknappung gelenkt und die Rolle der Ressourcen in den Hintergrund treten lassen (vgl. Krings \& Müller 2001). Bei dieser in der Neuen Institutionenökonomik prominenten Vorgehensweise werden aber die politischen Ordnungs- und Beziehungsstrukturen vernachlässigt und die institutionellen Regelungen werden funktionalistisch und statisch gesehen (Korf 2004; Ribot \& Peluso 2003). Ein weiterer und ebenso unterschätzter Aspekt, der vielleicht im Gegensatz zu anderen Naturressourcen besonders bei den Verfügungsrechten von Wasser eine bedeutende Rolle spielt, ist die symbolische Bedeutung von Wasser und seiner Konsumption. I. ILlich und E. SWyngedouw zeigen, inwiefern in Europa Wasser seit dem 18. Jahrhundert auch zur symbolischen sozialen Abgrenzung der Elite gegenüber der einfachen Bevölkerung diente. Von einer bis dahin herrschenden Hydrophobie ausgehend, gewinnt die nun mehr in der Privatsphäre verrichtete hydrophile Körperhygiene an Bedeutung und wird zum Zeichen einer sozialen Zugehörigkeit (ILLICH 1985; SwYNGEdouw 2005).

Im Fokus dieses Beitrages über den ungleichen Zugang zu Trinkwasser stehen die sozialen und politischen Beziehungen sowie die damit verbundene symbolische Bedeutung des Wassers. Die Untersuchung stützt sich dabei auf das relationale handlungstheoretische Analyseraster von Pierre Bourdieu, dessen theoretische Prämissen für die Geographie bereits an anderer Stelle erläutert wurden (vgl. u.a. DöRfLER et al. 2003; Hillier et al. 2002; LipPUNER 2005: 158ff.). Für die hier vorgestellten empirischen Ergebnisse und die Analyse der gesellschaftlichen Beziehungen soll für die vorliegende Argumentation lediglich das Konzept des Feldes herausgegriffen und kurz in Erinnerung gerufen werden:

Ein Feld ist aus objektiven Machtbeziehungen konstituiert und entsteht durch Beziehungen der Subjekte, die um Ressourcen, Positionen und Macht konkurrieren. Innerhalb dieses Feldes nehmen die Subjekte konkrete Positionen ein, die sie nur durch den Einsatz des ihnen verfügbaren Kapitals verändern können (Bourdieu \& WACQUANT 1992: 73). Dabei kann zwischen ökonomischen, sozialen, kulturellen und symbolischen Kapitaltypen unterschieden werden, die gegeneinander ausgetauscht werden können.

«Das Feld der sozialen Beziehungen wird damit durch die Versuche zur Aneignung und Maximierung verschiedener Sorten von Kapital konstituiert" (Dörfler et al. 2003: 16).

Das Feld stellt eine Struktur von objektiven Beziehungen zwischen Machtpositionen dar, die jene Strategien prägen und ausrichten, mit denen die Akteure versuchen, ihre Positionen zu halten oder auszubauen (BOURDIEU \& WaCQUANT 1992: 78).

Im Hohen Atlas sind wie in anderen ländlichen Räumen Marokkos Konflikte um Wasser an der Tagesordnung (siehe auch Stoffel et al. 2002). Während der Streit um Wasser im Bereich der Landwirtschaft über Generationen zu einem komplexen Regelwerk der Organisation von Bewässerung geführt hat (Hammoudi 1982; Jolly 1997; Ouhajou 1996), sind die Regeln für die Trinkwasserverteilung wenig kodifiziert, weil nach der islamischen Rechtsprechung alle das gleiche Recht auf Trinkwasser haben.

Im Untersuchungsgebiet, das im oberen Einzugsgebiet des Dra an der Südabdachung des Hohen Atlas liegt (Abb.1), nehmen die Konflikte um Trinkwasser an Häufigkeit und Intensität zu. Die Eigentumsansprüche an Quellen, die Zuschüttung von Brunnen und die gewalttätige Konfrontation zwischen mehreren Dörfern um das Nutzungsrecht einer Quelle haben im Juli 2001 zum Eingreifen von Vertretern des Zentralstaates und der Polizei geführt. So spektakulär diese Konflikte auch sein mögen, stellen sie doch nur den 


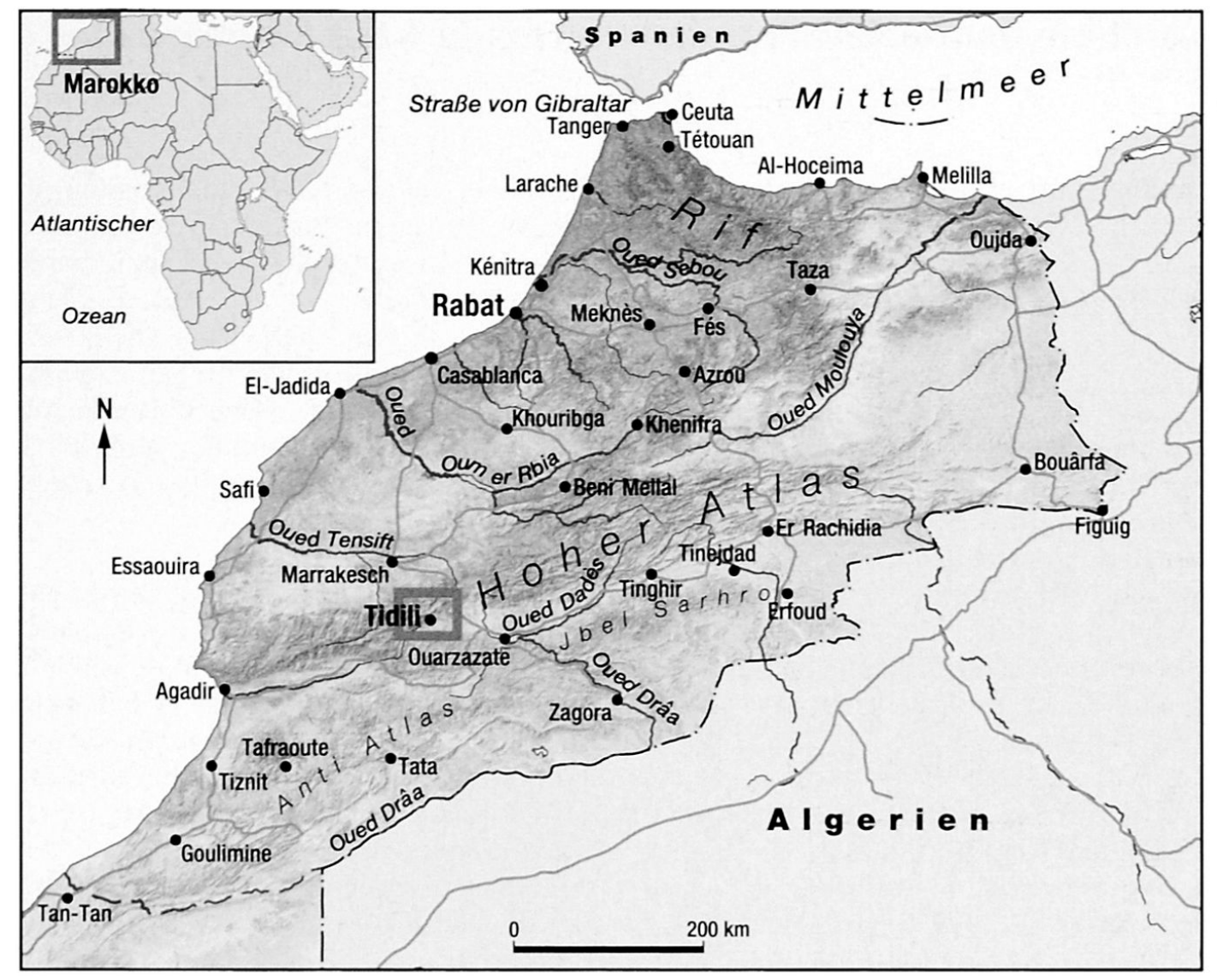

Abb. 1: Lage des Untersuchungsgebietes in Marokko

Location of the research area in Morocco

Localisation de la zone d'étude au Maroc

Kartengrundlage: ComputaMaps; Kartographie: M. WEGENER

sichtbaren Teil der Spannungen um die Wasserversorgung dar. Aufgrund der Dürreperiode, die zwischen 1998 und 2003 im Süden Marokkos registriert wurde (Haut Commissariat au Plan, HCP 2004), werden die beobachteten Konflikte um das Trinkwasser von den Beteiligten und Beobachtern als Folge der Verknappung gewertet.

Doch handelt es sich bei den anhaltenden Konflikten ausschließlich um ein Problem der unzureichenden Wassermenge, wenn die Trockenheit doch für dieses Gebiet am Nordrand der Sahara eigentlich charakteristisch ist? Welche Rolle spielen die wirtschaftlichen und gesellschaftlichen Veränderungen wie die Konsequenzen der Arbeitsmigration, der wachsende urbane Einfluss auf diesen peripheren Raum und die steigende Partizipation der Bevölkerung an lokalen Projekten?

Die folgenden Ausführungen stützen sich auf eine zwischen 2000 und 2004 durchgeführte Feldforschung in acht Dörfern, bei der sowohl teilnehmende Beob- achtung, qualitative Interviews und eine Befragung aller 185 Haushalte des Untersuchungsgebietes durchgeführt wurden. Im ersten Teil des Beitrags werden die Ungleichheiten im Trinkwasserzugang aufgezeigt. Anschließend wird dargestellt, inwiefern diese Disparitäten mit der aus der Kolonialzeit vererbten sozialen Hierarchie zusammenhängen und durch die Arbeitsmigration noch verstärkt werden. Abschließend soll geklärt werden, inwiefern die ungleiche Verteilung der Wasserversorgung aus den lokalen Machtverhältnissen resultiert.

\section{Die ungleiche Wasserversorgung der Dörfer}

Die Konflikte um Trinkwasser zwischen Dörfern des Untersuchungsgebietes sind weniger das Ergebnis einer Wasserverknappung als das Produkt eines ungleichen Ressourcenzugangs. Während die zwei Dörfer Imaghoudene und Aït Mérouane (unterer Teil) keine privaten Wasseranschlüsse besitzen, verfügen die meisten Haushalte der restlichen Siedlun- 


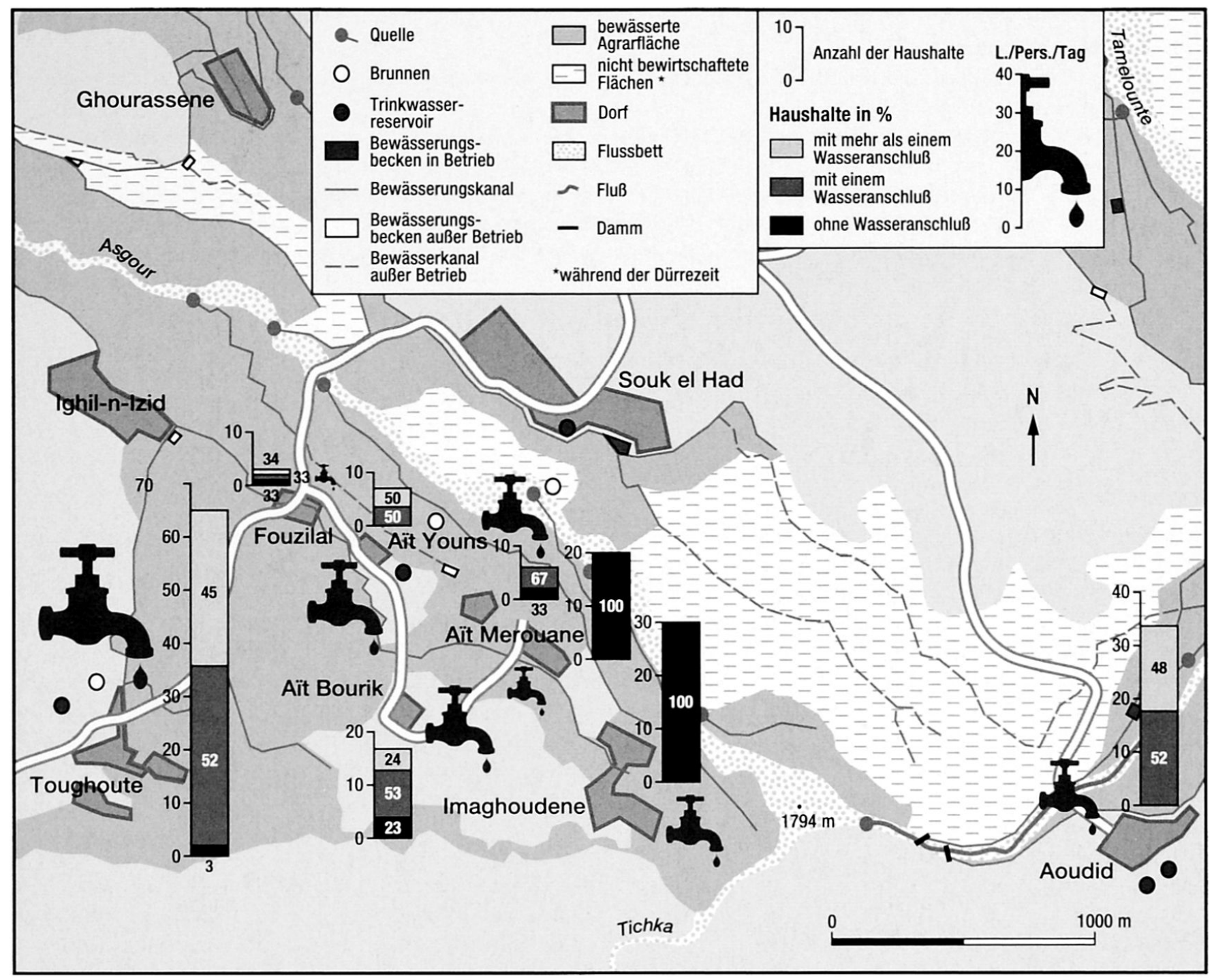

Abb. 2: Unterschiede bei Trinkwasserversorgung und -verbrauch in Tidili (2004)

Disparities in drinking water provision and consumption in Tidili (2004)

Les disparités d'approvisionnement et de consommation en eau potable à Tidili (2004)

Entwurf: O. Graefe; Kartographie: M. Wegener, O. Graefe

gen über mindesten einen Wasserhahn im Haus (vgl. Abb. 2). Die beiden genannten Dörfer besitzen weder einen Brunnen noch nutzen sie eine Quelle, die ein Wasserreservoir und ein zentrales Wasserverteilungsnetz speisen könnten. Dagegen werden Aït Youns, das obere Aït Mérouane, Aït Bourik und Toughoute über ein Wasserreservoir versorgt, nachdem die zuständige Gemeinde einen Brunnen und eine Pumpstation finanzierte. In diesen Dörfern haben jene Haushalte, die über die notwendigen finanziellen Mittel verfügen, einen Anschluss samt Zähler installiert, während sich die Ärmeren keinen eigenen Wasseranschluss leisten können. In Aoudid wurde bis zum Bau eines neuen Brunnens im Sommer 2004 die Versorgung von einer über dem Dorf gefassten Quelle und zwei Reservoirs sporadisch gesichert, was den relativ geringen Wasserkonsum erklärt (Abb. 2). Hier hat die Solidarität der Dorfbewohner auch den ärmsten Familien einen privaten Anschluss ermöglicht.

Die zwei nicht angeschlossenen Dörfer registrieren folglich einen weit geringeren Wasserverbrauch pro Person als die in den anderen Dörfern lebende Bevölkerung. Die Verbrauchsunterschiede zwischen den Dörfern mit einem zentralen Wasserversorgungssystem lassen sich durch die verschiedenen Wassertarife und die Einkommensunterschiede erklären. Die Grundgebühr von 5 Dirham (ca. 50 Eurocents) sowie der Preis pro Kubikmeter von 2,50 Dirham im Jahre 2004 ist in Toughoute um die Hälfte niedriger als in 


\section{Besitz oder Eigentum?}

Während das Eigentum die rechtliche Herrschaft über eine Sache bedeutet, beinhaltet der Besitz die tatsächliche Herrschaft darüber.

Im Fokus dieser Untersuchung stehen die tatsächliche Herrschaft über Land und nicht die Eigentumsverhältnisse, die sich im rechtspluralistischen Kontext des Hohen Atlas sehr unklar darstellen. In den abgelegenen Talschaften des Untersuchungsgebietes gibt es kein Bodenkataster, und vorislamisches, islamisches und staatliches Erbschaftsrecht existieren nebeneinander. Die befragten Besitzer sind in diesem Sinne nicht alle Grundeigentümer, sondern Nutznießer, sei es als Erbschaftsanwärter oder Pächter.

Kasten 1: Begriffserläuterung zu Besitz und Eigentum

Definition of ownership and property

Définition de la possession et de la propriété den anderen Dörfern. Toughoute ist gleichzeitig das Dorf, wo die reichsten Familien des Untersuchungsgebietes leben. Deren neue und mit Zement gebauten Häuser sind mit sanitären Einrichtungen ausgestattet (Wasseranschluss in der Küche, Duschen, Toilettenspülung), die einen höheren Wasserverbrauch bewirken. Dagegen besitzen die ärmeren Haushalte mit ihren in alter Lehmbautechnik gebauten Häusern meist nur einen Wasserhahn im Innenhof. Die unterschiedliche Ausstattung und der Verbrauch der Haushalte hängen somit mit ihrem jeweiligen sozioökonomischen Status zusammen.

\section{Eine wachsende soziale Differenzierung}

Diese kurze Beschreibung des Wasserkonsums, der Häuser und ihrer Ausstattung deutet bereits auf eine soziale Differenzierung innerhalb der Dorfgemeinschaften hin, die sich auch in den Landbesitzverhältnissen widerspiegelt (Kasten 1). Auch wenn sie nur einen Hinweis auf die reellen ökonomischen Ungleichheiten geben, kann man anhand ihrer räumlichen Verteilung eine Polarisierung feststellen (Abb. 3).

Die ungleiche Verteilung der landbesitzenden Familien, und vor allem der Familien ohne Land, ist auf die Sozialstruktur der Dörfer und ihre Geschichte zurückzuführen. Während die Erstsiedler von Toughoute, Imaghoudene und Aoudid sich die landwirtschaftlich günstigsten Flächen aneignen konnten, besteht die Bevölkerung des unteren Aït Mérouane aus ehemaligen Sklaven und Landarbeitern, die vor und während der Kolonialzeit für die Großbauern gearbeitet haben, zum Teil für den regionalen Fürsten und bedeutendsten Landbesitzer der Talschaft, den glaoui. Die seit etwa fünfzig Jahren in Aït Bourik lebende Bevölkerung besteht aus landlosen Landarbeitern und einfachen Handwerkern, die die Protektion einer einflussreichen Familie von islamischen Gelehrten gesucht haben.
Wenngleich diese Unterschiede bereits vor der französischen Protektoratszeit existierten (BERQUe 1978; MonTAGNE 1930), so wurden sie aufgrund der gewaltsamen Ausbeutung durch den glaoui El Thami in den Jahren zwischen 1930 und 1956 verstärkt. Die Zwangsarbeit und willkürlichen Enteignungen seiner Gegner sowie die materiellen Begünstigungen in Form von Arbeits- und Steuerbefreiungen seiner Anhänger (vgl. Amahan 1998; Le Prevost 1968) haben eine bis dahin unbekannte soziale Polarisierung bewirkt.

Die seit dem Protektorat erfolgten Arbeitsmigrationen haben die direkten Abhängigkeiten zwischen Landarbeitern und Landbesitzern teilweise abschwächen können, ohne dabei die sozioökonomischen Unterschiede zu mindern. Die seitdem von einer Minderheit ehemalig landloser Familien erworbenen Flächen reichen bei weitem nicht aus, um die Subsistenz dieser großen und zumeist Mehrgenerationenhaushalte zu sichern. Viele der Haushaltsvorstände sind daher gezwungen, andere Einkommensquellen zu suchen.

\section{Die durch die Arbeitsmigration verstärkte soziale Hierarchie}

Viele Autoren haben die durch Arbeitsmigrationen implizierten Veränderungen im Hohen Atlas untersucht (Aїт Hamza 1996, 1997a \& b; Amahan 1998; BüChNer 1986; Crépeau 1986; Fay 1986; TAMim 1993) und darauf hingewiesen, wie die besser gestellten Haushalte der Landbesitzer stärker an der Migration in die marokkanischen Städte und ins Ausland partizipieren und somit ihre sozioökonomischen Positionen im Vergleich zu den landlosen Familien weiter verbessern (Aḯ Hamza 2002; De HaAs 2003: 203f., 230f.).

Diese intensivere Einbindung der landbesitzenden Familien (vgl. Abb. 3) in die Migrationsprozesse bestä- 


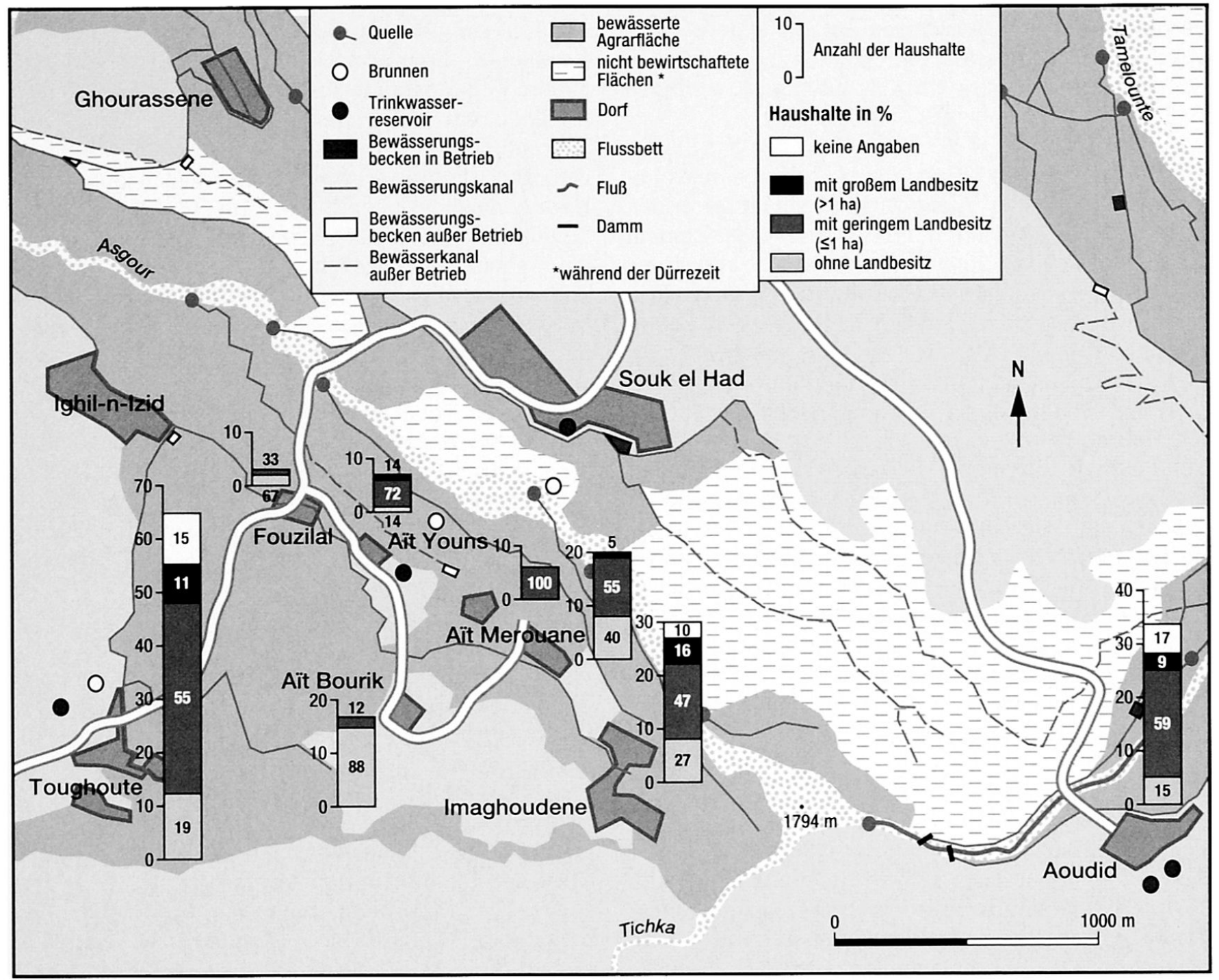

Abb. 3: Die Verteilung der Landbesitzverhältnisse im Untersuchungsgebiet (2004)

Distribution of land property rights in the research area (2004)

La distribution des droits de propriété fonciers dans la zone d'étude (2004)

Entwurf: O. Graefe; Kartographie: M. Wegener, O. Graefe

tigte sich auch im Fall des Untersuchungsgebietes, wo der Anteil der ökonomisch besser gestellten Haushalte mit Migranten in Toughoute, Ait Youns, Aoudid und Imaghoudene relativ hoch ist. Im Gegensatz dazu gibt es in Aït Mérouane und Aït Bourik, also eben dort, wo die Zahl der Landlosen und Kleinbauern am höchsten ist, zahlreiche Familien ohne Migranten.

Die Abwanderung eines oder mehrerer Familienmitglieder gibt noch keinen Hinweis darauf, ob und wie die im Dorf Zurückgebliebenen von diesen unterstützt werden und inwieweit die Migration die soziale Differenzierung verstärkt. Ohne die Einkommen der Migranten und Rimessen quantifizieren zu können, sollen aufgrund von zahlreichen Feldbeobachtun- gen und Interviews an dieser Stelle dennoch ein paar Tendenzen vorgestellt werden. Einerseits erhalten die Migranten aus den Familien mit großem Landbesitz (über einen Hektar) in der Regel höher qualifizierte und besser bezahlte Arbeitsstellen, während die Arbeitsmigranten aus ärmeren Familien sich eher mit einfacheren, schlecht bezahlten und unregelmäßigen Arbeiten begnügen müssen. Ein Grund dafür liegt in den Netzwerken, die von den reicheren Familien des Tidili mit der Stadt, insbesondere mit Marrakesch, teilweise über Generationen geknüpft worden sind. Neben unterschiedlichen Netzwerkzugängen kommt heute noch das ungleiche Engagement für die Schulbildung der Kinder hinzu. Landbesitzende Haushalte, die über das monetäre Einkommen eines Migranten 
verfügen, sind nicht gezwungen, auf die Feldarbeit der Kinder zurückzugreifen und können eine Strategie des sozialen Aufstiegs über Schulbildung verfolgen (vgl. Amahan 1998; De HaAs 2003).

Diese Unterschiede spiegeln sich auch in den Wohnformen und in der Ausstattung der Häuser wider. Während die Migranten ihre Häuser aus Zement bauen und sie mit sanitären Einrichtungen ausstatten, die denen eines Stadthauses keineswegs nachstehen, bleiben die ärmeren Familien in ihren alten Lehmhäusern. Es stellt sich die Frage, aus welchen Gründen die neuen, zum großen Teil überdimensionierten, dem Bergklima unangepassten und wenig komfortablen Häuser bevorzugt werden. M. Aïr Hamza (2002) deutet diese unrentable Bebauung auf dem Lande als eine Demonstration des sozialen Erfolgs und als Zeichen der sozialen Abgrenzung gegenüber jenen, die nicht erfolgreich sind oder nicht abwandern konnten. Die Fähigkeit, eine urbane Wohnform zur Schau stellen zu können, die neben dem Satellitenfernsehen, dem Telefon und der Möbelausstattung auch eine sanitäre Ausstattung beinhaltet, ist somit ein wichtiges symbolisches Zeichen für die soziale Differenzierung geworden (Aїт HAMZA 2002).

Die einflussreichen Familien folgen im Sinne der Konzeption Pierre Bourdieus (1980) somit einer Logik der Kapitalakkumulation unterschiedlicher Typen. Das ökonomische Kapital, hervorgebracht aus Landbesitz, Handel und Arbeitsmigration, versuchen diese Familien in soziales und symbolisches Kapital zu konvertieren, indem sie die - meist männlichen - Kinder in die Schule schicken und dem Familienoberhaupt die Pilgerfahrt nach Mekka finanzieren, und indem sie in Kulturgüter investieren wie das neue Haus und seine moderne Ausstattung. Die Akkumulation akzentuiert nicht nur die sozioökonomische Differenzierung, sondern stärkt auch die Position dieser Familien in der sozialen Hierarchie auf dörflicher und regionaler Ebene.

Dieser Prozess der sozialen Differenzierung, der von anderen Autoren bereits beschrieben (Aїт HAMZA 2002; De HaAs 2003) und hier nur kurz angeschnitten wurde, hat auch Folgen für den Zugang und Verbrauch von Trinkwasser auf der Haushalts- und Dorfebene. Dabei resultieren diese Disparitäten neben den Einkommensunterschieden auch aus politischen Entscheidungen.

\section{Lokale Machtverhältnisse und die Begünstigung bestimmter Dörfer}

Bezüglich der Unterschiede in Zugang und Konsum von Trinkwasser stellt sich die Frage, wie diese zu erklären sind. Warum verfügen manche Dörfer über Brunnen und ein zentrales Verteilungsnetz, während andere weder das eine noch das andere besitzen? Jene privilegierten Dorfgemeinschaften, die heute eine hydraulische Infrastruktur nutzen können, waren vor dem Bau der Wasserversorgungsanlagen durch ihre Lage keineswegs benachteiligt. Im Gegenteil besaßen Toughoute und Aoudid bereits seit mehreren Jahren ein Wasserverteilungsnetz, das aus gefassten Quellen oberhalb des Dorfes gespiesen wurde. Wenngleich die Leistung dieser Quellen stark schwankte und manchmal sogar zum Erliegen kam, profitierten die Frauen von einem bequemeren Zugang zu Trinkwasser. Die Frauen der benachbarten Siedlungen Imaghoudene und Aït Mérouane müssen mehrmals täglich das Wasser mit Kanistern und anderen Behältern aus weiter Entfernung holen. Die bereits in der Vergangenheit versorgten Dörfer haben somit ihre Situation verbessern können, wogegen die benachteiligte Dorfbevölkerung unterversorgt geblieben ist.

Die Begünstigung von Toughoute und Aït Youns sowie Aït Bourik und dem oberen Aït Mérouane folgt einer politischen Logik. Die für diese Wasserinfrastruktur verantwortliche Gemeinde versuchte durch die Maßnahmen, ihren mangelnden Einfluss auszugleichen. Dieses Legitimitätsdefizit kommt besonders in den Erzählungen und dem Verhalten der Dorfbevölkerung gegenüber den Gemeindevertretern zum Vorschein. Vorwürfe von Korruption, Veruntreuung öffentlicher Gelder, Wahlfälschung zugunsten bestimmter Gemeinderäte, willkürliche und parteiische Entscheidungen werden in Interviews und Gesprächen häufig genannt. Selbst wenn diese Anschuldigungen übertrieben sein mögen, so muss jedoch ein Bruch zwischen den Vertretern der Gemeinde und der Bevölkerung festgestellt werden. Ohne eine breite Unterstützung in der Bevölkerung und ohne breite soziale Basis greift die Leitung der Gemeinde auf eine klientelistische Strategie zurück. Sie bevorzugt die großen Dörfer, in denen die Familien mit einem höheren sozialen und symbolischen Kapital leben, und vor allem die Dörfer, die bisher gegenüber dem Gemeinderat und dem makhzen, d.h. dem Zentralstaat, Treue erwiesen haben. Die sozial unbedeutenden und kritischen Dorfgemeinschaften wurden bei den Entscheidungen der Gemeinde über die Infrastruktur nicht berücksichtigt. Die Entscheidung, zwei Brunnen und die Pumpstationen in Toughoute und Aït Youns zu installieren, zeugt von der Besorgnis der Gemeinde, die politische Unterstützung der reichen Familien zu behalten, eine Unterstützung, die sie verlieren könnte, wenn sie den anderen Dörfer den Vorrang geben würde.

Diese klientelistische Strategie erklärt allerdings nicht alleine die Unterschiede bei der dörflichen Trinkwasserversorgung. Sie entfaltet sich in einer lokal- 
politischen Konstellation, die sie begünstigt, denn die Dorfältesten und einflussreichen Familienoberhäupter profitieren von dieser Strategie. Sie können auf diese Weise ihren Einfluss geltend machen und somit ihre soziale Position stärken. Gegenüber Projekten, die von Mitgliedern der jüngeren Generation oder den unteren sozialen Schichten initiiert werden, zeigen sich die Dorfältesten oft skeptisch oder stellen sich ihnen entgegen. Hintergrund ist die Möglichkeit, dass die Initianten bei einer erfolgreichen Umsetzung dieser Projekte einen politischen Gewinn erzielen könnten. Indem sie sich gegen Infrastrukturprojekte wehren, versuchen einige Dorfälteste, ihre dominierende Position zu behalten und tragen somit zu den Disparitäten zwischen den Dörfern bei.

Die Vereine zur Dorfentwicklung (Associations locales de développement) werden meist von Jüngeren geleitet, die bereits migriert sind oder nach wie vor in den Städten arbeiten und von dort die Vereinsgeschäfte führen. Bereits von Fatima MERNissI beschrieben und, um ihre Fähigkeiten zur Selbsthilfe auszudrücken, als Aït Débrouille bezeichnet (2003), existieren diese Dorfvereine in der Talschaft des Tidili nunmehr in jedem Dorf und sind im Bereich der Wasserversorgung sehr aktiv. Das zentrale Trinkwasserverteilungsnetz vor dem Bau der Pumpstation in Aoudid wurde z.B. von dem lokalen Entwicklungsverein finanziert und gebaut. Doch der Erfolg dieser Vereine hängt neben der oben genannten Position der Dorfältesten auch von der Logik und Motivation ab, mit der diese Vereine arbeiten. Zweifelsohne sind die meisten Mitglieder aus Engagement für das Heimatdorf aktiv. Einige beteiligen sich aber auch aus politischem Interesse an der Vereinsarbeit. In einem ländlichen Kontext, wo Parteipolitik noch wenig akzeptiert ist, stellen die Vereine für sie ein Instrument der politischen Auseinandersetzung dar, mit dessen Hilfe sie gegen die bestehende Ordnung angehen können. Die Vereine unterliegen dabei einer Substitutionslogik gegenüber den bisherigen lokalen und öffentlichen Machtinstanzen wie dem Dorfrat (j'mâa), die weiter an Einfluss verlieren.

Für andere sind die Vereine ein Mittel, um an die Gelder der Entwicklungshilfe zu gelangen. Deren oft vermutete und manchmal festgestellte Veruntreuung ist Grund für den Austritt oder das Desinteresse vieler Mitglieder und sogar für die Opposition der Dorfbevölkerung gegenüber manchen Projekten wie in Aoudid und Imaghoudene.

Die unterschiedliche Mobilisierungs- und Durchsetzungsfähigkeit der Vereine trägt, nebst den Machtverhältnissen zugunsten der Dörfer, in denen die einflussreichsten Familien mit einem hohen sozialen Kapital leben, zu einer ungleichen Trinkwasserversorgung bei.

\section{Fazit}

Die Konflikte um Wasser im Untersuchungsgebiet sind weniger das Ergebnis von Wasserknappheit, sondern resultieren viel mehr aus einem ungleichen Zugang zu Trinkwasser. Diese Konflikte, die manchmal offen und gewalttätig ausgetragen werden wie im Juli 2001 zwischen Aoudid, Imaghoudene und Aït Mérouane, sind um so virulenter, als sich die benachteiligte Bevölkerung sehr wohl bewusst ist, dass ihre Situation keineswegs naturgegeben ist. Die Disparitäten in der Wasserversorgung sind das Produkt einer sozialen Hierarchie und politischer Machtverhältnisse.

Diese Machtverhältnisse zwischen den alten und neuen Akteuren, zwischen den Dorfältesten und der Gemeinde, zwischen Vertretern unterschiedlicher Generationen und sozialer Gruppen begünstigen bestimmte Dörfer zum Nachteil anderer. Die Entscheidungen über den Bau der Infrastruktur resultieren aus diesen sozialen und politischen Beziehungen und verstärken sie zugleich.

Im Sinne von Illich (1985) und Swyngedouw (2004) dienen auf der Haushaltsebene die sanitären Einrichtungen und der Wasserkonsum zur Darstellung einer urbanen Lebensweise, die sich vom Bauerntum und von den sozioökonomisch Schwächeren absetzt. Darüber hinaus ermöglichen sie eine Bestätigung der über die Abstammung geerbten dominanten Position, Legitimität und Macht. Für jene Migranten, die nicht aus relativ wohlhabenden Familien stammen, bedeutet der Hausanschluss nicht nur eine Verbesserung der Wasserversorgung, sondern auch eine Möglichkeit, soziale Anerkennung zu erreichen. Dadurch können sie politisch stärker partizipieren, was ihnen aufgrund ihrer Herkunft bisher verwehrt war. Die Versorgungsverbesserung und die höhere Konsumption stellen somit Versuche dar, ökonomisches Kapital in soziales und schließlich in politisches Kapital umzusetzen. Der Zugang zu Trinkwasser und die Form der Versorgung sind somit in doppelter Hinsicht Gegenstände von Konflikten: als Instrument der symbolischen Dominanz für die einen und als Mittel zur sozialen Anerkennung für die anderen.

Die Konflikte resultieren aus dynamischen Machtverhältnissen und weniger aus unwirtlichen und unsicheren meteorologischen und hydrologischen Bedingungen. Die Wasserknappheit und der Zugang zu Trinkwasser werden vielmehr als Plattform benutzt, soziale Konflikte auszutragen, die an anderer Stelle nur schwer zum Ausdruck kommen. Die auf unterschiedlichen Maßstabsebenen existierenden Diskurse der Medien, der öffentlichen Verwaltung, der Dorfältesten und der Gemeinde über die «Dürre» und ihre stetige Präsenz verschleiern politische Entscheidun- 
gen und Verantwortlichkeiten. Sie tragen dazu bei, ein Problem zu entpolitisieren und zu naturalisieren, das der politischen und weniger der natürlichen Sphäre zuzuordnen ist.

\section{Dank}

Der Deutschen Forschungsgemeinschaft (DFG) sei für die finanzielle Unterstützung dieser Arbeit im Rahmen des Projekts «Trinkwasserverteilung im Hohen Atlas: sozialer Wandel und Konflikte» gedankt.

\section{Literatur}

Aïт HamZA, M. (1996): Emigration et formations socioéconomiques au sud de l'Atlas. Cas du douar Amjgag. - In: Le bassin du Dra: carrefour civilisationnel et espace de culture et de création. $-=$ Publications de la Faculté des Lettres et des Sciences Humaines d'Agadir, Agadir: 61-71.

Aïr Hamza, M. (1997a): Auswirkungen der Arbeitsmigration auf die Oasen in Südmarokko. - In: Geographische Rundschau 49: 83-88.

Aїт Hamza, M. (1997b): La migration et les transformations sociales dans un village sud-marocain: Amajgag. - In: Migration internationale et changements sociaux dans le Maghreb. Colloque international de Hammamet, Tunisie, 21-25 juin 1993. - = Série: Colloques 7, Faculté des Sciences Humaines et Sociales de Tunis, Tunis: 381-403.

Aïr Hamza, M. (2002): Mobilité socio-spatiale et développement local au sud de l'Atlas marocain (DadésTodgha). - = Maghreb-Studien 13, Passau.

Amahan, A. (1998): Mutations sociales dans le HautAtlas. Les Ghoujdama. - Paris: Editions de la Maison des sciences de l'homme, Rabat: Editions la Porte.

AyeB, H. (1998): L'Eau au Proche-Orient: la guerre n'aura pas lieu. - Paris: Khartala/Kairo: Centre d'Etudes et de Documentation Economique, Juridique et Sociale (CEDEJ).

BERQUe, J. (1978): Structures sociales du Haut-Atlas. - Paris: Presses Universitaires de France.

Bourdieu, P. (1980): Le sens pratique. - Paris: Editions de Minuit.

Bourdieu, P. \& L.J.D. Wacquant (1992): Réponses. Pour une anthropologie réflexive. - Paris: Seuil.

BüchNeR, H.-J. (1986): Die temporäre Arbeitskräftewanderung nach Westeuropa als bestimmender Faktor für den gegenwärtigen Strukturwandel der TodrhaOase (Südmarokko). - = Mainzer geographische Studien 18, Mainz.

Bulloch, J. \& A. Darwish (1993): Water wars. Coming conflicts in the Middle East. - London: Gollancz.

CrépeAu, C. (1986): Mutations sociales et spatiales dans l'Ounein et le pays Id Daoud ou Ali. - In: Revue de l'Occident musulman et de la Méditerranée 41-42: 249-263.
Direction de la statistique, Royaume du Maroc (Hrsg.) (2004): Annuaire statistique du Maroc. - Rabat: Direction de la statistique.

Diop, S. \& P. Rekacewicz (2003): Atlas mondial de l'eau. - Paris: Editions Autrement.

Dörfler, T., Graefe, O. \& D. Müller-Mahn (2003): Habitus und Feld. Anregungen für eine Neuorientierung der geographischen Entwicklungsforschung auf der Grundlage von Bourdieus «Theorie der Praxis». - In: Geographica Helvetica 58, 1: 11-23.

FAY, G. (1986): Désagrégation des collectivités et dégradation des milieux dans le Haut-Atlas marocain. - In: Revue de l'Occident musulman et de la Méditerranée 41-42: 234-248.

HAAS, H. DE (2003): Migration and development in Southern Morocco. The disparate socio-economic impacts of out-migration on the Todgha Oasis Valley. - Rotterdam: Optima Grafische Comjunicatie.

Hammoudi, A. (1982): Droits d'eau et société. La vallée du Dra. - In: Hommes, Terres \& Eaux 12: 105-118.

Haut Commissariat au Plan (2005/6): Annuaire Statistique du Maroc 2004. - Rabat.

Hillier, J. \& E. Rooksby (Hrsg.) (2002): Habitus: a sense of place. - Burlington: Ashgate.

ILLICH, I. (1985): $\mathrm{H}^{2} \mathrm{O}$ and the waters of forgetfulness: reflections on the historicity of «stuff». - Dallas: Dallas Institute of Humanities and Culture.

JolLY, G. (1997): La maîtrise lignagère de l'irrigation dans la vallée de l'Azzaden (Haut-Atlas, Maroc). Vision historique et spatiale. - In: Berque, J., Garrigues-Cresswell, B., Jolly, G. \& B. Lecestre-RolLIER (Hrsg.): Jacques Berque. La Méditerranée, le Haut-Atlas. Etudes réunies par Christian Bromberger. - Aix-en-Provence: Publications de l'Université de Provence: 59-86.

Korf, B. (2004): Conflict, space and institutions. Property rights and the political economy of war in Sri Lanka. - Aachen: Shaker.

Krings, T. \& B. Müller (2001): Politische Ökologie: theoretische Leitlinien und aktuelle Forschungsfelder. - In: Reuber, P. \& G. Wolkersdorfer: Politische Geographie. Handlungsorientierte Ansätze und Critical Geopolitics. - = Heidelberger geographische Arbeiten 112, Heidelberg: 93-116.

LASSERRE, F. (1999): Le prochain siècle sera-t-il celui des guerre de l'eau? - In: Revue internationale et stratégique 33 , Paris.

LASSERre, F. (2003): L'eau, enjeu mondial - géopolitique du partage de l'eau. - Paris: Le Serpent à Plumes.

Le Prévost, J. (1968): El Glaoui. - Paris: Editions du Dialogue.

Lippuner, R. (2005): Raum Systeme Praktiken. Zum Verhältnis von Alltag, Wissenschaft und Geographie. - Stuttgart: Franz Steiner Verlag.

Mernissi, F. (2003): ONG rurales du Haut-Atlas. Les Aitt-Débrouille. - Rabat: Marsam. 
Montagne, R. (1930): Les Berbères et le Makhzen dans le sud du Maroc. - Paris: Alcan.

Mutin, G. (2000): L'eau dans le monde arabe. - Paris: Ellipses.

OpP, C. (2004): Probleme des Wasserdargebots und der Wassernutzung im 21. Jahrhundert. - In: Marburger geographische Schriften 140: 6-21.

OuhaJou, L.(1996): Espace hydraulique et société au Maroc. Cas des systèmes d'irrigation dans la vallée du Dra. - Thèses et mémoires 7, Publications de la Faculté des Lettres et des Sciences Humaines d'Agadir.

Ribot, J.C. \& N.L. Peluso (2003): A theory of access. - In: Rural Sociology 68, 2: 153-181.

SEN, A. (1981): Poverty and famines. An essay on entitlements and deprivation. - Oxford: Clarendan.

SOFFER, A. (1999): Rivers of fire. The conflict over water in the Middle East. - Lanham: Rowman \& Littlefield.

StARR, J. (1991): Water wars. - In: Foreign policy 82: 17-36.

Stoffel, M., Monbaron, M. \& D. Maselli (2002): Mountains and lowlands: enemies or partners? Example of the High Atlas, Morocco. - Fribourg: University of Fribourg.

Swyngedouw, E. (2004): Social power and the urbanization of water: flows of power. - Oxford, New York: Oxford University Press.

TамIM, M. (1993): L'émigration internationale de travail dans l'Ouneine, Haut-Atlas du Toubkal. - In: Migration internationale et changements sociaux dans le Maghreb, Colloque international de Hammamet, Tunisie. - = Série: Colloques 7, Faculté des Sciences Humaines et Sociales de Tunis, Tunis: 433-451.

United Nations Environmental Programme (2002): Vital water graphics. - http://www.unep.org/vitalwater/ 13-freshwater-availabil.html 25.1.2006.

\section{Zusammenfassung: Wasser, Konflikte und soziales Kapital im Hohen Atlas Südmarokkos}

In diesem Beitrag über den ungleichen Zugang zu Trinkwasser stehen die sozialen und politischen Beziehungen sowie die damit verbundene symbolische Bedeutung des Wassers im Mittelpunkt. Auf Basis der relationalen Handlungstheorie von Bourdieu wird gezeigt, wie Wasser als Mittel verwendet wird, soziale Differenzierung symbolisch auszudrücken beziehungsweise anzufechten. Nach einer kurzen Darstellung der mit der sozialen Hierarchie verbundenen Unterschiede in der Wasserversorgung und im Wasserverbrauch werden die Hintergründe und die mit den politischen Beziehungen verknüpfte Trinkwasserverteilung analysiert. Die Verbesserung der Wasserversorgung und die höhere Konsumption werden dabei als ein Konvertierungsversuch von ökonomischem Kapital in soziales Kapital gedeutet. Aus dieser analytischen Perspektive erscheinen die zum Teil gewalttätigen Konflikte um die dörfliche Wasserversorgung weniger als Folge der Wasserknappheit, sondern vielmehr als Ausdruck politischer Auseinandersetzungen zwischen sozialen Gruppen.

\section{Résumé: Eau, conflits et capital social dans le Haut Atlas au sud du Maroc}

Cette contribution sur l'accès inégal à l'eau potable est centrée sur les rapports sociaux et politiques ainsi que sur le sens symbolique de l'eau qui y est étroitement associé. Fondée sur la théorie de l'action de Bourdieu, l'analyse montre comment l'eau est utilisée comme moyen d'expression et de contestation symbolique des inégalités sociales. La distribution de l'eau est analysée dans son contexte politique après avoir esquissé les disparités d'approvisionnement et de consommation d'eau potable qui peuvent être mises en rapport avec la hiérarchie sociale. L'amélioration de l'approvisionnement en eau et sa consommation accrue sont ainsi interprétées comme une tentative de convertir le capital économique en capital social. Dans cette perspective analytique, les conflits, parfois violents, pour l'approvisionnement des villages ne sont pas présentés uniquement comme une conséquence de la sécheresse, mais également comme l'expression de tensions politiques entre groupes sociaux.

\section{Summary: Water, conflict and social capital in the Higher Atlas Mountains of South Morocco}

The focus in this article is on inequality of access to drinking water, with special attention being paid to related social and political relationships and the associated symbolism of water. Based on Bourdieu's Relational Theory of Action, the analysis shows how water is used as a means of symbolic expression and contestation of social inequalities. The water distribution is analysed in its political context after a brief overview of the disparities in drinking water provision and consumption related to social hierarchy. The improvement of water provision and the thereby induced increase in water consumption are interpreted as an attempt to convert economic capital into social capital. The partly violent conflicts related to the village drinking water supply appear in this light to be more an expression of the political struggle between social groups than a consequence of the drought.

Dr. Olivier Graefe, Lehrstuhl für Bevölkerungs- und Sozialgeographie, Universität Bayreuth, Universitätsstrasse 30, D-95447 Bayreuth.

e-mail: Olivier.Graefe@uni-bayreuth.de

\section{Manuskripteingang/received/manuscrit entré le} 3.11.2005

Annahme zum Druck/accepted for publication/accepté pour l'imperession: 28.3 .2006 